\title{
Análise Comparativa de Segurança e Eficácia entre as Vias de Acesso Radial e Femoral na Realização de Intervenção Coronária Percutânea no Infarto Agudo do Miocárdio
}

\author{
José Ary Boechat e Salles ${ }^{1,2,3}$, Júlio César Machado Andrea ${ }^{1,2}$, Leandro Assumpção Cortes², \\ Lilian Vieira Carestiato', Luis Felipe Camillis dos Santos ${ }^{1}$, Hélio Roque Figueira ${ }^{1,2,3}$
}

\section{RESUMO}

Introdução: A intervenção coronária percutânea é a estratégia mais adequada de reperfusão no infarto agudo do miocárdio. A intervenção coronária percutânea pela via radial nas síndromes estáveis é uma alternativa efetiva, comparativamente ao acesso pela via femoral, com redução do risco de complicações hemorrágicas e vasculares. Este estudo teve como objetivo avaliar a segurança e a efetividade da intervenção coronária percutânea pela via radial no infarto agudo do miocárdio em uma série consecutiva de pacientes. Método: Entre janeiro de 1997 e julho de 2009, 387 pacientes foram tratados por intervenção coronária percutânea nas primeiras 12 horas de infarto agudo do miocárdio, sendo excluídos os pacientes em choque cardiogênico e aqueles submetidos a angioplastia de ponte de safena e angioplastia com balão. Foram realizadas 132 angioplastias transluminais coronárias por via radial $(34,8 \%)$ e 247 por via femoral $(65,2 \%)$. Resultados: Obteve-se elevado sucesso angiográfico (95,6\% vs. 96,8\%; $P=0,57)$, com o mesmo porcentual de perfusão miocárdica pós-procedimento, independentemente da via de acesso utilizada. Aos 30 dias de evolução, observou-se tendência a menor incidência de eventos no grupo em que foi utilizada a via radial $(1,5 \%$ vs. $6,9 \% ; \mathrm{P}=0,07)$, possivelmente pela menor ocorrência de trombose do stent nesse grupo $(0,8 \%$ vs. $4,9 \% ; P=0,04)$ e pela menor necessidade de nova revascularização percutânea do vaso-alvo $(0,8 \%$ vs. $4,9 \% ; P=0,04)$. Não foram observadas diferenças na incidência de hemorragias maiores entre os dois grupos $(2,3 \%$ vs. $2 \%$; P > 0,99). Conclusões: $O$ presente estudo sugere que o acesso pela via radial na angioplastia primária em pacientes elegíveis é seguro e eficaz quando o procedimento é realizado por intervencionistas "radialistas", sendo uma alternativa atrativa ao acesso femoral, especialmente naqueles pacientes com alto risco de com-

\section{ABSTRACT}

\author{
Safety and Efficacy of Radial Versus Femoral \\ Approach in Percutaneous Coronary Intervention \\ in Acute Myocardial Infarction
}

Background: Percutaneous coronary intervention $(\mathrm{PCI})$ is the most appropriate reperfusion strategy in acute myocardial infarction. $\mathrm{PCl}$ by the radial approach in stable coronary syndromes is an effective alternative when compared to the femoral approach, with reduced risk of bleeding and vascular complications. This paper is aimed at evaluating the safety and efficacy of $\mathrm{PCl}$ in acute myocardial infarction by the radial approach in a series of consecutive patients. Method: From January 97 to July 2009, 387 patients were treated by $\mathrm{PCl}$ within 12 hours of acute myocardial infarction. Patients with cardiogenic shock and those undergoing balloon angioplasty or saphenous vein graft $\mathrm{PCl}$ were excluded. One hundred and thirty-two $\mathrm{PCl}$ by radial approach $(34.8 \%)$ and 247 by femoral approach $(65.2 \%)$ were carried out. Results: High angiographic success $(95.6 \%$ vs. $96.8 \% ; P=0.57$ ) was observed with the same rate of post-procedure myocardial perfusion, regardless of the approach used. After 30 days, there was a trend towards lower incidence of events in the radial approach group $(1.5 \%$ vs. $6.9 \% ; P=0.07)$, possibly due to the lower occurrence of stent thrombosis in this group $(0.8 \%$ vs. $4.9 \% ; \mathrm{P}=0.04)$ and less need of target vessel revascularization $(0.8 \%$ vs. $4.9 \%$; $P=0.04)$. There was no difference in the incidence of major bleeding between the two groups $(2.3 \%$ vs. $2 \% ; P>0.99)$. Conclusions: The present study suggests that the radial approach in primary angioplasty is safe and effective when performed by interventional "radialists" in eligible patients, and is an attractive alternative to the femoral approach, especially in patients at high risk of vascular complications such as

\footnotetext{
Clínica São Vicente - Rio de Janeiro, RJ, Brasil.

2 Hospital CardioTrauma - Rio de Janeiro, RJ, Brasil.

3 Hospital de Clínicas de Niterói - Niterói, RJ, Brasil.

Correspondência: José Ary Boechat e Salles. Rua João Borges, 204 -

Gávea - Rio de Janeiro, RJ, Brasil - CEP 22451-100

E-mail: josearyboechat@gmail.com

Recebido em: 7/10/2009 • Aceito em: 13/12/2009
} 
Boechat e Salles JA, et al. Análise Comparativa de Segurança e Eficácia entre as Vias de Acesso Radial e Femoral na Realização de Intervenção Coronária Percutânea no Infarto Agudo do Miocárdio. Rev Bras Cardiol Invas. 2009;17(4):498-504.

plicações vasculares associadas à via de acesso, como os obesos e os que recebem inibidores da glicoproteína Ilb/IIla e trombolíticos.

DESCRITORES: Infarto do miocárdio. Stents. Angiografia coronária. Artéria radial. Artéria femoral. Angioplastia transluminal percutânea coronária. Resultado de tratamento.

A angioplastia coronária percutânea é a estratégia ideal para recanalização da artéria coronária culpada no cenário de infarto agudo do miocárdio ${ }^{1}$. Seus resultados agudo e a longo prazo são otimizados pelo implante de stents e por regimes farmacológicos agressivos, com antiplaquetários e anticoagulantes ${ }^{2,3}$.

Desde a introdução da intervenção coronária pela via radial por Kiemeneij e Laarman ${ }^{4}$, seu uso tem sido cada vez mais popularizado ${ }^{5,6}$ em decorrência da baixa incidência de complicações no sítio de punção arterial e do aumento do conforto do paciente, quando comparada à intervenção coronária transfemoral’ . A redução do tamanho dos introdutores arteriais para cateteres $6 \mathrm{~F}$, associada à evolução do perfil dos cateteres balão, tornou a via radial uma técnica segura para realização de intervenções coronárias eletivas ${ }^{8}$.

Os dados sobre a eficácia da abordagem por via radial no tratamento do infarto agudo do miocárdio são limitados ${ }^{9-11}$, sendo inclusive critério de exclusão para alguns estudos ${ }^{7}$. Sua maior vantagem é a quase ausência de complicações vasculares significativas, mesmo em pacientes de alto risco, como obesos, hipertensos, mulheres e especialmente aqueles em uso de trombolíticos e inibidores da glicoproteína IIb/IIla.

A abordagem por via radial, entretanto, tem sido criticada por consumir mais tempo, com retardo da reperfusão por dificuldades técnicas em obter acesso vascular e/ou canulação das coronárias ${ }^{12,13}$, e pela menor taxa de sucesso quando comparada à abordagem transfemoral ${ }^{14}$. Além disso, possui outras limitações, como dificuldade em utilizar cateteres mais calibrosos (como o $8 \mathrm{~F}$ ), balão intra-aórtico e marcapassos temporários.

Este estudo teve como objetivo avaliar a segurança e a eficácia da via de acesso radial no tratamento do infarto agudo do miocárdio, numa série consecutiva não-randomizada de pacientes tratados nas primeiras 12 horas de evolução, comparados a pacientes tratados pela via femoral, no período de 1997 a 2009.

\section{MÉTODO}

Estudo retrospectivo utilizando banco de dados de uma série consecutiva de pacientes tratados por angioplastia coronária nas 12 horas iniciais do infarto obese patients and those receiving glycoprotein IIb/IIla inhibitors or thrombolytics.

DESCRIPTORS: Myocardial infarction. Stents. Coronary angiography. Radial artery. Femoral artery. Angioplasty, transluminal, percutaneous coronary. Treatment outcome.

agudo do miocárdio em três hospitais privados do Rio de Janeiro (RJ), entre 1997 e 2009, por apenas uma equipe de cardiologistas intervencionistas. Foram excluídos da análise pacientes tratados somente por angioplastia por balão, choque cardiogênico e oclusão de enxerto de safena. A decisão da via de acesso para angiografia ficou a critério do operador, guiada por fatores tais como calibre da artéria radial, experiência e fatores relacionados ao paciente, como peso, estado clínico e uso de inibidores de glicoproteína IIb/IIla.

\section{Via radial}

Para permitir acesso rápido e seguro, aplicou-se o teste de Allen para verificação do duplo suprimento sanguíneo da mão, com preparo simultâneo do acesso pela via femoral, utilizado em caso de insucesso ou demora na punção radial ou para uso de marcapasso provisório ou balão intra-aórtico, quando necessário. Após anestesia local, era realizada punção da artéria radial com a colocação de introdutor longo, seguida de injeção intra-arterial de solução de heparina 10.000 UI e $10 \mathrm{mg}$ de mononitrato de isossorbida para prevenção de espasmo. Após o procedimento, a bainha era retirada na sala de hemodinâmica, sendo feito curativo compressivo.

\section{Via femoral}

A canulação da artéria femoral foi feita conforme técnica padrão de Judkins, com inserção de introdutor 6 F. A hemostasia ao fim do procedimento foi realizada após a retirada da bainha na própria sala de hemodinâmica, com dispositivos de oclusão arterial ou, mais frequentemente, por compressão manual na Unidade Coronária após normalização do tempo de coagulação ativada.

\section{Angioplastia primária}

Independentemente do sítio de acesso escolhido, as angioplastias foram realizadas utilizando-se preferencialmente cateter-guia $6 \mathrm{~F}$, com ultrapassagem da obstrução com corda-guia 0,014, seguida de dilatação com balão de troca rápida e implante de stent. O sucesso primário foi definido como fluxo distal TIMI 3 ao término do procedimento, com estenose residual inferior a 20\% (análise visual). O sangramento maior foi definido como aquele associado a queda da hemoglobina superior a $5 \mathrm{~g} / \mathrm{dl}$ ou do hematócrito acima de 
$15 \%$, necessidade de intervenção cirúrgica, transfusão sanguínea ou sangramento intracraniano. Eventos cardíacos maiores foram definidos como ocorrência de óbito (cardíaco ou não), reinfarto ou necessidade de nova revascularização do vaso-alvo nos primeiros 30 dias após o procedimento.

\section{Análise estatística}

As variáveis contínuas foram expressas como média \pm desvio padrão e comparadas pelo teste $t$ de Student. As variáveis categóricas foram expressas como números absolutos e porcentuais e comparadas com os testes de qui-quadrado ou exato de Fisher, quando necessário. Foram considerados significantes valores de $\mathrm{P}<0,05$.

\section{RESULTADOS}

No período de janeiro de 1997 a julho de 2009, foram realizadas 4.598 intervenções coronárias percutâneas, das quais 1.787 (38,8\%) por via radial. Foram tratados 379 pacientes com angioplastia primária e implante de stents nas primeiras 12 horas de evolução, sendo $132(34,8 \%)$ tratados por via radial e os demais, por via femoral. Até julho de 2001, a única via de acesso utilizada no tratamento do infarto agudo do miocárdio era a femoral; atualmente, a via radial é utilizada em quase dois terços desses procedimentos.

As características demográficas e clínicas foram, em geral, similares, com predomínio de pacientes do sexo masculino (73\%), média de idade de 60 anos e $15 \%$ de diabéticos (Tabela 1). Os pacientes abordados pela via radial apresentaram-se para o tratamento mais tardiamente $(7,5 \pm 4,1$ horas vs. 5,2 \pm 3,4 horas para os grupos radial e femoral, respectivamente; $P<0,001)$, mas em melhor classificação funcional Killip-Kimball. O tempo porta-balão foi semelhante entre os dois grupos (54,2 \pm 31,2 minutos vs. $53,3 \pm 19,5$ minutos para os grupos radial e femoral, respectivamente; $\mathrm{P}=0,73)$.

As características angiográficas e do procedimento (Tabela 2) também não apresentaram diferenças, com metade dos pacientes exibindo acometimento uniarterial e quase dois terços, função ventricular normal ou com discreta alteração da contratilidade. O grupo tratado por via radial, entretanto, demonstrou maior porcentual de vasos com fluxo epicárdico pré-angioplastia transluminal coronária TIMI $>0$. Observou-se maior utilização do acesso por via femoral em infartos nãoanteriores, possivelmente pela necessidade do uso de marca-passo provisório, de mais fácil execução quando realizado por via femoral. Nos últimos dois anos, no entanto, o implante de marca-passo provisório por punção de veia superficial na altura da prega cubital no mesmo braço utilizado para acesso da artéria radial tem reduzido nossa utilização da via femoral nos infartos não-anteriores. Foram implantados 172 stents por via radial (1,3 3 0,6 stent/paciente) e 331 stents por via femoral (1,3 \pm 0,6 stent/

TABELA 1

Características demográficas e clínicas

\begin{tabular}{|c|c|c|c|}
\hline Variável & $\begin{array}{c}\text { Radial } \\
(n=132)\end{array}$ & $\begin{array}{l}\text { Femoral } \\
(n=247)\end{array}$ & $\mathbf{P}$ \\
\hline Média de idade, anos & $60,2 \pm 12$ & $60,8 \pm 13$ & 0,66 \\
\hline Sexo masculino, n (\%) & $103(78)$ & $175(70,9)$ & 0,14 \\
\hline \multicolumn{4}{|l|}{ Fatores de risco, n (\%) } \\
\hline Diabetes & $22(16,7)$ & 37 (15) & 0,66 \\
\hline Hipertensão arterial & $94(71,2)$ & $135(54,7)$ & 0,002 \\
\hline Tabagismo & $46(34,8)$ & $82(33,2)$ & 0,82 \\
\hline Hipercolesterolemia & $86(65,2)$ & $119(48,2)$ & 0,001 \\
\hline Insuficiência renal, n (\%) & $6(4,5)$ & $6(2,4)$ & 0,35 \\
\hline Infarto prévio, n (\%) & $7(5,3)$ & $21(8,5)$ & 0,31 \\
\hline CRM prévia, n (\%) & 0 & $3(1,2)$ & 0,55 \\
\hline ATC prévia, n (\%) & $12(9,1)$ & $27(10,9)$ & 0,72 \\
\hline Classe Killip-Kimball & & & 0,05 \\
\hline I & $119(90,2 \%)$ & $200(81 \%)$ & \\
\hline II & $10(7,6 \%)$ & $41(16,6 \%)$ & \\
\hline III & $3(2,3 \%)$ & $6(2,4 \%)$ & \\
\hline Delta $T$, horas & $7,5 \pm 4,1$ & $5,2 \pm 3,4$ & $<0,001$ \\
\hline Tempo porta-balão, minutos & $54,2 \pm 31,2$ & $53,3 \pm 19,5$ & 0,73 \\
\hline
\end{tabular}

ATC = angioplastia transluminal coronária; CRM = cirurgia de revascularização miocárdica; $\mathrm{n}$ = número de pacientes. 
TABELA 2

Características angiográficas e do procedimento

\begin{tabular}{|c|c|c|c|}
\hline Variável & $\begin{array}{c}\text { Radial } \\
(n=132 / 137 \text { vasos })\end{array}$ & $\begin{array}{c}\text { Femoral } \\
(n=247 / 252 \text { vasos })\end{array}$ & $\mathbf{P}$ \\
\hline Extensão da doença coronária, n (\%) & & & 0,66 \\
\hline 1 vaso & $55(41,7)$ & $123(49,8)$ & \\
\hline 2 vasos & $53(40,2)$ & $68(27,5)$ & \\
\hline 3 vasos & $24(18,2)$ & $56(22,7)$ & \\
\hline Função ventricular esquerda, n (\%) & & & 0,44 \\
\hline Normal & $9(6,8)$ & $24(9,7)$ & \\
\hline Leve & $79(59,8)$ & $120(48,6)$ & \\
\hline Moderada & $39(29,5)$ & $93(37,7)$ & \\
\hline Grave & $5(3,8)$ & $10(4)$ & \\
\hline Vaso tratado, n (\%) & & & 0,01 \\
\hline Tronco de coronária esquerda & $2(1,5)$ & $1(0,4)$ & \\
\hline Descendente anterior & $66(48,5)$ & $101(40,1)$ & \\
\hline Circunflexa & $26(19,1)$ & $32(12,7)$ & \\
\hline Coronária direita & $42(30,9)$ & $118(46,8)$ & \\
\hline Classificação das lesões, n (\%) & & & 0,18 \\
\hline A & 0 & $4(1,6)$ & \\
\hline B1 & $36(26,3)$ & $56(22,2)$ & \\
\hline B2 & $60(43,8)$ & $144(57,1)$ & \\
\hline $\mathrm{C}$ & $41(29,9)$ & $48(19)$ & \\
\hline Fluxo epicárdico pré-ATC, n (\%) & & & 0,03 \\
\hline TIMI 0 & $92(69,7)$ & $198(80,2)$ & \\
\hline TIMI 1 & $8(6,1)$ & $8(3,2)$ & \\
\hline TIMI 2 & $6(4,5)$ & $10(4)$ & \\
\hline TIMI 3 & $26(19,7)$ & $31(12,6)$ & \\
\hline Perfusão miocárdica pós-ATC, n (\%) & & & 0,34 \\
\hline Blush 0 & 0 & $2(1,3)$ & \\
\hline Blush 1 & $5(4,1)$ & $5(3,3)$ & \\
\hline Blush 2 & $28(23,1)$ & $42(27,6)$ & \\
\hline Blush 3 & $88(72,7)$ & $103(67,8)$ & \\
\hline ATC em vaso não-alvo, n (\%) & $5(3,8)$ & $5(2)$ & 0,33 \\
\hline Número de stents/paciente & $1,3 \pm 0,6$ & $1,3 \pm 0,6$ & $>0,99$ \\
\hline Inibidor da glicoproteína IIb/IIla, n (\%) & $49(37,1)$ & $79(32)$ & 0,36 \\
\hline Sucesso angiográfico, n (\%) & $131(95,6)$ & $244(96,8)$ & 0,57 \\
\hline No reflow, $\mathrm{n}(\%)$ & $10(7,3)$ & $15(6)$ & 0,66 \\
\hline
\end{tabular}

paciente), tendo sido utilizados inibidores da glicoproteína IIb/IIla em um terço dos procedimentos $(\mathrm{P}=0,36)$. O sucesso angiográfico foi elevado e semelhante entre os grupos $(95,6 \%$ vs. $96,8 \%$ para os grupos radial e femoral, respectivamente; $\mathrm{P}=0,57)$. O período de hospitalização foi menor na via radial (4,6 dias vs. 5,4 dias para os grupos radial e femoral, respectivamente; $\mathrm{P}<0,001)$.

Observou-se menor incidência de eventos nos primeiros 30 dias de evolução nos pacientes tratados por via radial $(1,5 \%$ vs. $6,9 \% ; P=0,07)$, possivelmente pela menor ocorrência de trombose do stent nesse grupo $(0,8 \%$ vs. $4,9 \%$; $P=0,04)$ e pela menor necessidade de nova revascularização percutânea do vasoalvo $(0,8 \%$ vs. $4,9 \%$; $=0,04)$ (Tabela 3$)$. Entretanto, não foi observada diferença na mortalidade entre os grupos $(0,8 \%$ vs. $2 \%$ para os grupos radial e femoral, respectivamente; $P=0,66$ ), assim como não foram observadas diferenças entre os grupos quanto à incidência de hemorragias maiores $(2,3 \%$ vs. $2 \%$ para os grupos radial e femoral, respectivamente; $\mathrm{P}>0,99$ ). 
TABELA 3

Eventos clínicos aos 30 dias

\begin{tabular}{lccc}
\hline & $\begin{array}{c}\text { Radial } \\
(\mathbf{n = 1 3 2})\end{array}$ & $\begin{array}{c}\text { Femoral } \\
(\mathbf{n = 2 4 7})\end{array}$ & $\mathbf{P}$ \\
\hline ECAM, $\mathrm{n}(\%)$ & $2(1,5)$ & $17(6,9)$ & 0,07 \\
Óbito & $1(0,8)$ & $5(2)$ & 0,66 \\
$\quad$ Reinfarto & $1(0,8)$ & $12(4,9)$ & 0,04 \\
Nova ATC & $1(0,8)$ & $12(4,9)$ & 0,04 \\
CRM & 0 & $3(1,2)$ & 0,55 \\
Sangramento maior, $\mathrm{n}(\%)$ & $3(2,3)$ & $5(2)$ & $>0,99$ \\
$\quad$ Sítio de acesso & 0 & $2(0,8)$ & \\
Digestivo & $1(0,8)$ & 0 & \\
Cerebral & $2(1,5)$ & 0 & 0,54 \\
Pseudoaneurisma & 0 & $3(1,2)$ & 0,71 \\
Sangramento menor, $\mathrm{n}(\%)$ & 0 & $2(0,8)$ & 0,04 \\
Trombocitopenia, $\mathrm{n}(\%)$ & $1(0,8)$ & $3(1,2)$ & $12(4,9)$ \\
Trombose do stent, $\mathrm{n}(\%)$ & $1(0,8)$ & $1 \%$ & \\
\hline
\end{tabular}

ATC = angioplastia transluminal coronária; CRM = cirurgia de revascularização miocárdica; ECAM = eventos cardíacos adversos maiores; $\mathrm{n}=$ número de pacientes.

\section{DISCUSSÃO}

Em 1983, Hartzler et al. ${ }^{15}$ foram os primeiros a descrever o potencial benefício da estratégia de reperfusão mecânica no infarto agudo, com o objetivo de restaurar o fluxo coronário anterógrado na artéria relacionada ao infarto. Atualmente, a angioplastia primária é a melhor abordagem disponível ${ }^{16}$, apesar dos desafios logísticos, estando associada a altas taxas de sucesso primário, eliminando a estenose coronária subjacente e restaurando o fluxo coronário normal.

Entretanto, as complicações hemorrágicas no sítio de punção arterial femoral (2,5\% a $11 \%)^{17,18}$ podem agravar e até limitar o beneficio clínico da angioplastia primária, especialmente quando antiplaquetários e antitrombínicos são utilizados. A ocorrência de sangramento maior está associada a aumento do risco de eventos, incluindo morte e recorrência dos eventos isquêmicos em vários estudos ${ }^{19,20}$. Os prováveis mecanismos incluem consumo de proteínas anticoagulantes, aumento do turnover de plaquetas, e efeitos adversos induzidos pela hemotransfusão e interrupção precoce dos antitrombóticos/anticoagulantes ${ }^{8}$.

A artéria radial é teoricamente um sítio de acesso seguro, pois é um vaso superficial, isolado de nervos e veias, de rápido acesso, permitindo hemostasia fácil e eficiente. A viabilidade e a eficácia da angioplastia por via radial no tratamento de lesões coronárias têm sido amplamente documentadas em vários cenários clínicos ${ }^{21}$, especialmente nas angioplastias coronárias não-emergenciais, com baixíssima incidência de complicações vasculares, permitindo deambulação precoce e maior conforto do paciente e da equipe clínica, além da alta hospitalar precoce ${ }^{22}$. O beneficio é ampliado em pacientes idosos, em obesos e naqueles em uso de antiplaquetários e anticoagulantes.

A reduzida incidência de complicações hemorrágicas no sítio de acesso após a utilização da via radial nas angioplastias eletivas impulsionou os afeitos à técnica a empregá-la nos paciente com infarto agudo ${ }^{9-11,23}$.

Dificuldade de aprendizado da técnica e reduzido calibre da artéria radial são geralmente as maiores limitações da intervenção pela via radial, quando comparada à técnica femoral. Sem experiência suficiente, o acesso pela via radial é mais difícil, com maior exposição a radiação, tornando o procedimento mais longo e sem outros benefícios, exceto a menor incidência de complicações no sítio de acesso ${ }^{13}$. A curva de aprendizado mais longa pode ter impacto negativo especialmente nos pacientes com infarto, nos quais o atraso na reperfusão está associado a redução da sobrevida.

Nossa experiência com coronariografia diagnóstica por via radial foi iniciada em 1997, tendo sido realizadas, desde então, mais de 5 mil coronariografias por essa via, sendo atualmente a via de escolha para a realização de procedimentos diagnósticos (> 95\%), inclusive com utilização da artéria radial esquerda para estudo nos pacientes revascularizados. Os procedimentos terapêuticos pela via radial representam, atualmente, cerca de $70 \%$ das intervenções não-relacionadas ao infarto agudo e $60 \%$ das angioplastias primárias.

Os achados do presente estudo sugerem que a angioplastia coronária transradial no infarto agudo realizada por intervencionistas treinados na técnica radial é tão eficaz quanto a angioplastia por via femoral, 
Boechat e Salles JA, et al. Análise Comparativa de Segurança e Eficácia entre as Vias de Acesso Radial e Femoral na Realização de Intervenção Coronária Percutânea no Infarto Agudo do Miocárdio. Rev Bras Cardiol Invas. 2009;17(4):498-504.

com elevada taxa de sucesso angiográfico. A ocorrência de complicações vasculares foi baixa, independentemente da via de acesso utilizada, ocorrendo em apenas $2,8 \%$ dos pacientes tratados pela via femoral, o que pode, em parte, ser explicado pelo menor uso de inibidores de glicoproteína nesse grupo. Os dispositivos de oclusão vascular femoral foram utilizados em menos de $5 \%$ dos casos. O tempo porta-balão, a taxa de reperfusão e a restauração do fluxo epicárdico normal não foram influenciados pela via de acesso escolhida, com baixa ocorrência de eventos maiores nos primeiros 30 dias de evolução.

\section{LIMITAÇÕES}

Trata-se de estudo retrospectivo de centro único, com análise de banco de dados derivado da rotina clínica e não de estudo controlado. A escolha do sítio de acesso pode ter sido ditada pelo estado clínico do paciente, com os pacientes de maior risco sendo tratados pela via femoral, o que pode ter influenciado a ocorrência de eventos cardíacos maiores. Apesar desse viés de seleção, os pacientes tratados por via radial não apresentaram complicações no sítio de acesso, o que tem sido demonstrado por outros estudos.

\section{CONCLUSÕES}

O presente estudo sugere que a utilização da via radial para angioplastia primária em pacientes elegíveis é segura e eficaz quando realizada por intervencionistas "radialistas", sendo uma alternativa atrativa ao acesso femoral, especialmente naqueles pacientes com alto risco de complicações vasculares associadas à via de acesso, como os obesos e os que recebem inibidores da glicoproteína Ilb/Illa e trombolíticos.

\section{CONFLITO DE INTERESSES}

Os autores declararam inexistência de conflito de interesses.

\section{REFERÊNCIAS BIBLIOGRÁFICAS}

1. Weaver WD, Simes RJ, Betriu A, Grines CL, Zijlstra F, Garcia $E$, et al. Comparison of primary coronary angioplasty and intravenous thrombolytic therapy for acute myocardial infarction: a quantitative review. JAMA. 1997;278(23):2093-8. Erratum in: JAMA. 1998;279(23):1876. Comment in: ACP J Club. 1998;128(3):59. JAMA. 1997;278(23):2110-1.

2. Grines CL, Cox DA, Stone GW, Garcia E, Mattos LA, Giambaertolomei A, et al. Coronary angioplasty with or without stent implantation for acute myocardial infarction. Stent Primary Angioplasty in Myocardial Infarction Study Group. N Engl J Med. 1999;341(26):1949-56. Comment in: N Engl J Med. 2000;342(19):1447; author reply 1448. N Engl J Med. 1999;341(26):2005-6. N Engl J Med. 2000; 342(19):1447-8. N Engl J Med. 2000;342(19):1448.

3. Tcheng JE, Kandzari DE, Grines $C L$, Cox DA, Effron $M B$, Garcia E, Griffin JJ, Guagliumi G, Stuckey T, Turco M, Fahy M, Lansky AJ, Mehran R, Stone GW; CADILLAC Investigators. Benefits and risks of abciximab use in primary angioplasty for acute myocardial infarction: the Controlled Abciximab and Device Investigation to Lower Late Angioplasty Complications (CADILLAC) Trial. Circulation. 2003;108(11):1316-23.

4. Kiemeneij F, Laarman GJ. Percutaneous transradial artery approach for the coronary artery stent implantation. Cathet Cardiovasc Diagn. 1993;30(2):173-8. Erratum in: Cathet Cardiovasc Diagn. 1993;30(4):358.

5. Saito S, Miyake S, Hosokawa G, Tanaka S, Kawamitsu K, Kaneda $\mathrm{H}$, et al. Transradial coronary intervention in Japanese patients. Cathet Cardiovasc Interv. 1999;46(1):37-41; discussion 42.

6. Saito S. Transradial approach-from the evangelist's view. Cathet Cardiovasc Interv. 2001;53(2):269-70. Comment in: Cathet Cardiovasc Interv. 2001;53(2):264-8.

7. Kiemeneij F, Laarman GJ, Odekerken D, Slagboom T, van der Wieken R. A randomized comparison of percutaneous transluminal coronary angioplasty by the radial, brachial and femoral approaches: the Access study. J Am Coll Cardiol. 1997;29(6):1269-75.

8. Jolly SS, Amlani S, Hamon M, Yusuf S, Mehta SR. Radial versus femoral access for coronary angiography or intervention and the impact on major bleeding and ischemic events: a systematic review and meta-analysis of randomized trials. Am Heart J. 2009;15(1):132-40.

9. Ochiai M, Isshiki T, Toyoizumi H, Eto K, Yokoyama N, Koyama $\mathrm{Y}$, et al. Efficacy of transradial primary stenting in patients with acute myocardial infarction. Am J Cardiol. 1999;83(6):966-8.

10. Mathias DW, Bigler L. Transradial coronary angioplasty and stent implantation in acute myocardial infarction: initial experience. J Invasive Cardiol. 2000;12(11):547-9. Comment in: J Invasive Cardiol. 2000;12(11):553-4. J Invasive Cardiol. 2000;12(11):550-2.

11. Kim MH, Cha KS, Kim HJ, Kim SG, Kim JS. Primary stenting for acute myocardial infarction via the transradial approach: a safe and useful alternative to the transfemoral approach. J Invasive Cardiol. 2000;12(6):292-6. Comment in: J Invasive Cardiol. 2000;12(6):297.

12. Lotan C, Hasin Y, Mosseri M, Rozenman Y, Admon D, Nassar $\mathrm{H}$, et al. Transradial approach for coronary angiography and angioplasty. Am J Cardiol. 1995;76(3):164-7.

13. Benit E, Missault L, Eeman T, Carlier M, Muyldermans L, Materne $\mathrm{P}$, et al. Brachial, radial, or femoral approach for elective Palmaz-Schatz stent implantation: a randomized comparison. Cathet Cardiovasc Diagn. 1997;41(2):124-30.

14. Lotan C, Hasin Y, Mosseri M, Rozenman Y, Admon D, Nassar $\mathrm{H}$, et al. Transradial approach for coronary angiography and angioplasty. Am J Cardiol. 1995;76(3):164-7.

15. Hartzler GO, Rutherford BD, McConahay DR, Johnson WL Jr, McCallister BD, Gura GM Jr, et al. Percutaneous transluminal coronary angioplasty with and without thrombolytic therapy for treatment of acute myocardial infarction. Am Heart J. 1983;106(5 Pt 1):965-73.

16. Keeley EC, Boura JA, Grines CL. Primary angioplasty versus intravenous thrombolytic therapy for acute myocardial infarction: a quantitative review of 23 randomised trials. Lancet. 2003; 361(9351):13-20. Comment in: ACP J Club. 2003;139(1):1. Lancet. 2004;364(9439):1014-5. Lancet. 2003;361(9365): 1303; author reply 1304-5. Lancet. 2003;361(9361):967; author reply 967-8. Lancet. 2003;361(9365):1304; author reply 1304-5. Lancet. 2003;361(9361):965-6; author reply 967-8. Lancet. 2003;361(9361):966; author reply 967-8. Lancet. 2003; 361(9365):1303-4; author reply 1304-5.

17. Stone GW, Brodie BR, Griffin JJ, Morice MC, Costantini C, St. Goar FG, et al. Prospective, multicenter study of the safety and feasibility of primary stenting in acute myocardial infarction: in-hospital and 30-day results of the PAMI stent pilot trial. Primary Angioplasty in Myocardial Infarction Stent Pilot Trial Investigators. J Am Coll Cardiol. 1998;31(1):23-30. 
Boechat e Salles JA, et al. Análise Comparativa de Segurança e Eficácia entre as Vias de Acesso Radial e Femoral na Realização de Intervenção Coronária Percutânea no Infarto Agudo do Miocárdio. Rev Bras Cardiol Invas. 2009;17(4):498-504.

18. Mahdi NA, Lopez J, Leon M, Pathan A, Harrell L, Jang IK, et al. Comparison of primary coronary stenting to primary balloon angioplasty with stent bailout for the treatment of patients with acute myocardial infarction. Am J Cardiol. 1998;81(8):957-63.

19. Chase A, Fretz E, Warburton W, Klinke WP, Carere RG, Pi $D$, et al. Association of the arterial access site at angioplasty with transfusion and mortality: the M.O.R.T.A.L study (Mortality benefit Of Reduced Transfusion after percutaneous coronary intervention via the Arm or Leg). Heart. 2008;94(8):1019-25. Comment in: Heart. 2008;94(12):1530-2.

20. Eikelboom J, Mehta SR, Anand SS, Xie C, Fox KA, Yusuf S. Adverse impact of bleeding on prognosis in patients with acute coronary syndromes. Circulation. 2006;114(8):774-82.
21. Agostoni $\mathrm{P}$, Biondi-Zoccai GG, de Benedictis ML, Rigattieri S, Turri M, Anselmi M, et al. Radial versus femoral approach for percutaneous coronary diagnostic and interventional procedures; systematic overview and meta-analysis of randomized trials. J Am Coll Cardiol. 2004;44(2):349-56.

22. Jabara R, Gadesam R, Pendyala L, Chronos N, Crisco LV, King SB, et al. Ambulatory discharge after transradial coronary intervention: Preliminary US single-center experience (Sameday TransRadial Intervention and Discharge Evaluation, the STRIDE Study). Am Heart J. 2008;156(6):1141-6.

23. Tebet MA, Andrade PB, Gentile M, Mattos LA, Labrunie A. Angioplastia primária via radial com doses aumentadas de tirofiban: uma técnica segura e efetiva. Rev Bras Cardiol Invasiva. 2007;15(3):255-60. 